\title{
THE HOLOMORPHIC KERNEL OF THE RANKIN-SELBERG CONVOLUTION*
}

\author{
DORIAN GOLDFELD ${ }^{\dagger}$ AND SHOUWU ZHANG ${ }^{\ddagger}$
}

1. Introduction. Fix positive integers $k, \ell, N, D$. Let $S_{k}\left(\Gamma_{0}(N)\right)$ denote the $\mathbb{C}$-vector space of holomorphic cusp forms of weight $k$ for the congruence subgroup

$$
\Gamma_{0}(N)=\left\{\left(\begin{array}{ll}
a & b \\
c & d
\end{array}\right) \in S L(2, \mathbb{Z}) \mid c \equiv 0(\bmod N)\right\} .
$$

For a Dirichlet character $\epsilon$ of $(\mathbb{Z} / D \mathbb{Z})^{\times}$, let $M_{\ell}\left(\Gamma_{0}(D), \epsilon\right)$ denote the $\mathbb{C}$-vector space of holomorphic modular forms of weight $\ell$ with character $\epsilon$ for the congruence group $\Gamma_{0}(D)$

Let $f \in S_{k}\left(\Gamma_{0}(N)\right)$ and $g \in M_{\ell}\left(\Gamma_{0}(D), \epsilon\right)$ have Fourier expansions of the form

$$
f(z)=\sum_{n=1}^{\infty} a(n) n^{\frac{k-1}{2}} e^{2 \pi i n z}, \quad g(z)=b(0)+\sum_{n=1}^{\infty} b(n) n^{\frac{\ell-1}{2}} e^{2 \pi i n z} .
$$

Rankin and Selberg $[\mathbf{R}]$, [S1] proved that the convolution L-function (in the case of equal weights $k=\ell$ )

$$
L(s, f \otimes g)=\sum_{n=1}^{\infty} \frac{a(n) b(n)}{n^{s}}
$$

converges absolutely for complex $s$ with $\operatorname{Re}(s)>1$, has a meromorphic continuation in $s$ with at most a simple pole at $s=1$, and satisfies a functional equation $s \rightarrow 1-s$. This result was later generalized $[\mathbf{L}]$ to more general situations, and in particular, to arbitrary pairs of weights $k, \ell$.

The proof of the meromorphic continuation and the functional equation of $L(s, f \otimes g)$ was obtained by expressing $L(s, f \otimes g)$ as an inner product of $f \cdot g$ with a nonholomorphic Eisenstein series. We shall give a new proof of this result which does not use Eisenstein series at all, but instead expresses the Rankin-Selberg convolution L-function as an inner product of $f$ with a holomorphic kernel function which depends on $g$ and $s$. The main result of the paper is the Fourier expansion of the kernel function (when $D$ is squarefree) which is given in Theorem 6.5. In the case where $\epsilon$ is a quadratic Dirichlet character $(\bmod D)$, a simpler and more explicit version of this result is given in Theorem 9.1. The functional equation of the kernel is stated and proved in various important cases in sections $\S 10, \S 11$.

In the special case that $g$ is a theta function attached to the imaginary quadratic extension $\mathbb{Q}(\sqrt{-D})$, the value of the holomorphic kernel function (or its derivative) at $s=\frac{1}{2}$ coincides with the kernel function computed by Gross and Zagier $[\mathbf{G}-\mathbf{Z}]$ in their celebrated formula relating the derivative of an L-function of an elliptic curve with the height of a certain Heegner point. Thus, our method simultaneously gives a new simplified proof of the L-value computation in the Gross-Zagier formula together with

\footnotetext{
* Received July 15, 1999; accepted for publication August 31, 1999.

$\dagger$ Department of Mathematics, Columbia University, New York City, NY. 10027, U.S.A. (dg@ math.columbia.edu).

$\ddagger$ Department of Mathematics, Columbia University, New York City, NY. 10027, U.S.A. (szhang @math.columbia.edu).
} 
a new proof of the meromorphic continuation and functional equation of the RankinSelberg convolution. The original method of Gross-Zagier used non-holomorphic Eisenstein series defined on a smaller group. The kernel was then obtained by a trace map and a holomorphic projection. In our method, all calculations are done directly on $S_{k}\left(\Gamma_{0}(N)\right)$ and it is not necessary to go outside the holomorphic space with different level and then project back in later.

2. Poincaré Series. For $\gamma=\left(\begin{array}{ll}a & b \\ c & d\end{array}\right) \in S L(2, \mathbb{Z})$ let

$$
j(\gamma, z)=c z+d
$$

denote the one-cocycle which satisfies $j\left(\gamma \cdot \gamma^{\prime}, z\right)=j\left(\gamma, \gamma^{\prime} z\right) \cdot j\left(\gamma^{\prime}, z\right)$ for all matrices $\gamma, \gamma^{\prime} \in S L(2, \mathbb{Z})$. Fix positive integers $m, k$. For $\operatorname{Re}(s)>1-\frac{k}{2}$, the series

$$
P_{m}(z, s)=m^{\frac{k-1}{2}} \sum_{\gamma \in \Gamma_{\infty} \backslash \Gamma_{0}(N)} e^{2 \pi i m \gamma z} j(\gamma, z)^{-k}(\operatorname{Im} \gamma z)^{s} .
$$

converges absolutely and uniformly to an automorphic form of weight $k$ on $\Gamma_{0}(N)$. This series was first introduced by Selberg [S2] and shown to have a meromorphic continuation to the entire complex $s$-plane. We define the holomorphic Poincaré series

$$
P_{m}(z)=\lim _{s \rightarrow 0} P_{m}(z, s)
$$

by analytic continuation.

Fourier Expansion: The Fourier coefficients $p_{m}(n)$ of

$$
P_{m}(z)=\sum_{n=1}^{\infty} p_{m}(n) n^{\frac{k-1}{2}} e^{2 \pi i n z}
$$

are given by the formula (see [Sa]),

$$
p_{m}(n)=\delta_{m, n}+2 \pi i^{k} \sum_{\substack{c=1 \\ c \equiv 0}}^{\infty} \frac{S(m, n ; c)}{c} J_{k-1}\left(\frac{4 \pi \sqrt{m n}}{c}\right)
$$

where $\delta_{m, n}$ (Kronecker's delta function) is 1 if $m=n$ and zero otherwise; $S(m, n ; c)$ is the Kloosterman sum

$$
S(m, n ; c)=\sum_{a d \equiv 1(\bmod c)} e^{\frac{2 \pi i}{c}(m a+n d)}
$$

and

$$
J_{k-1}(y)=\frac{1}{2 \pi i} \int_{\epsilon-\frac{k-1}{2}-i \infty}^{\epsilon-\frac{k-1}{2}+i \infty} \frac{\Gamma\left(\frac{k-1}{2}+w\right)}{\Gamma\left(\frac{k+1}{2}-w\right)}\left(\frac{y}{2}\right)^{-2 w} d w
$$

is the Bessel function.

Petersson Formula By unfolding the integral, one can show that for any cusp form

$$
h(z)=\sum_{m=1}^{\infty} c(m) m^{\frac{k-1}{2}} e^{2 \pi i m z} \in S_{k}\left(\Gamma_{0}(N)\right),
$$


we have

$$
c(m)=\frac{(4 \pi)^{k-1}}{(k-2) !}<h, P_{m}>.
$$

3. Outline of the Method. Let $(\cdot, \cdot)$ denote the Weil-Petersson inner product on $S_{k}\left(\Gamma_{0}(N)\right)$. Now, fix $s \in \mathbb{C}$ and

$$
g=b(0)+\sum_{n=1}^{\infty} b(n) n^{\frac{\ell-1}{2}} e^{2 \pi i n z} \in M_{\ell}\left(\Gamma_{0}(D), \epsilon\right) .
$$

Consider the linear map

$$
f \longrightarrow L(s, f \otimes g)=\left\langle\Phi_{s, g}, f>\right.
$$

for a unique holomorphic Riesz kernel (cusp form) $\Phi_{s, g} \in S_{k}\left(\Gamma_{0}(N)\right)$ with Fourier expansion

$$
\Phi_{s, g}(z)=\sum_{n=1}^{\infty} \phi_{s, g}(n) n^{\frac{k-1}{2}} e^{2 \pi i n z}
$$

We now use the properties of the Poincaré series (Fourier expansion and Petersson formula) to obtain a formula for the Fourier coefficients of the Riesz kernel $\Phi_{s, g}$ given in formula (3.2).

Set $h=\Phi_{s, g}$ in formula (2.4). It immediately follows from (3.1) that

$$
\phi_{s, g}(m)=\frac{(4 \pi)^{k-1}}{(k-2) !} L\left(s, P_{m} \otimes g\right) .
$$

By the Fourier expansion (2.1) for the Poincaré series, we have for complex $s$ with $R e(s)>1+\frac{k-1}{2}$ that

$$
L\left(s, P_{m} \otimes g\right)=b(m) m^{-s}+2 \pi i^{k} T_{m}(s)
$$

where

$T_{m}(s)=\sum_{\substack{c=1 \\ N \mid c}}^{\infty} \sum_{n=1}^{\infty} \frac{S(m, n: c)}{c} \frac{b(n)}{n^{s}} \cdot \frac{1}{2 \pi i} \int_{\epsilon-\frac{k-1}{2}-i \infty}^{\epsilon-\frac{k-1}{2}+i \infty} \frac{\Gamma\left(\frac{k-1}{2}+w\right)}{\Gamma\left(\frac{k+1}{2}-w\right)}\left(\frac{2 \pi \sqrt{m n}}{c}\right)^{-2 w} d w$

$$
=\sum_{\substack{c=1 \\ N \mid c}}^{\infty} \sum_{r \in(\mathbb{Z} / c \mathbb{Z})^{\times}} e^{\frac{2 \pi i m r}{c}} \cdot \frac{1}{2 \pi i} \int_{\epsilon-\frac{k-1}{2}-i \infty}^{\epsilon-\frac{k-1}{2}+i \infty} \frac{\Gamma\left(\frac{k-1}{2}+w\right)}{\Gamma\left(\frac{k+1}{2}-w\right)} \frac{(2 \pi \sqrt{m})^{-2 w}}{c^{1-2 w}} L_{g}\left(s+w, \frac{\bar{r}}{c}\right) d w,
$$

and

$$
L_{g}\left(s, \frac{r}{c}\right)=\sum_{n=1}^{\infty} b(n) e^{\frac{2 \pi i n r}{c}} n^{-s}
$$

with $r \bar{r} \equiv 1(\bmod c)$.

In the remainder of this section we briefly illustrate our method in the special case $N=D=1, k=\ell$, and $g$ is a cusp form. Complete details for the more general case are given in $\S 4$ through $\S 10$. 
Our assumptions imply that in this case $L_{g}\left(s, \frac{a}{c}\right)$ has holomorphic continuation to all $s \in \mathbb{C}$ and satisfies the functional equation (see Proposition 4.2)

$$
L_{g}\left(s, \frac{a}{c}\right)=i^{-k}\left(\frac{c}{2 \pi}\right)^{1-2 s} \frac{\Gamma\left(\frac{k+1}{2}-s\right)}{\Gamma\left(\frac{k-1}{2}+s\right)} L_{g}\left(1-s,-\frac{\bar{a}}{c}\right)
$$

where $\bar{a}$ is the inverse of $a(\bmod c)$. If we apply this functional equation to the formula for $T_{m}(s)$, given in (3.5), we obtain

$$
T_{m}(s)=\frac{(2 \pi)^{2 s-1}}{i^{k}} \sum_{n=1}^{\infty} \frac{b(n)}{n^{1-s}} S(s, m-n) I_{s}\left(\frac{m}{n}\right)
$$

where

$$
S(s, B)=\sum_{c=1}^{\infty} \frac{1}{c^{2 s}} \sum_{r \in(\mathbb{Z} / c \mathbb{Z})^{\times}} \exp \left(\frac{2 \pi i B}{c} r\right),
$$

is the classical Ramanujan sum, and

$$
I_{s}(y)=\frac{1}{2 \pi i} \int_{\epsilon-\frac{k-1}{2}-i \infty}^{\epsilon-\frac{k-1}{2}+i \infty} \frac{\Gamma\left(\frac{k-1}{2}+w\right) \Gamma\left(\frac{k+1}{2}-s-w\right)}{\Gamma\left(\frac{k+1}{2}-w\right) \Gamma\left(\frac{k-1}{2}+s+w\right)} y^{-w} d w
$$

is a hypergeometric function.

Formula for $S(s, B)$ : The formula

$$
S(s, B)=\frac{1}{\zeta(2 s)} \sum_{d \mid B} d^{1-2 s}
$$

was first given by Ramanujan [Ra]. When $B=0$,

$$
S(s, 0)=\frac{\zeta(2 s-1)}{\zeta(2 s)}=\pi^{2 s-3 / 2} \frac{\Gamma(1-s)}{\Gamma\left(s-\frac{1}{2}\right)} \frac{\zeta(2(1-s))}{\zeta(2 s)} .
$$

Formula for $I_{s}(x)$ : We will show in Proposition 8.3 that

$$
I_{s}(x)= \begin{cases}\frac{\Gamma(k-s)}{\Gamma(k) \Gamma(s)} x^{\frac{k-1}{2}}(1-x)^{s-1} F\left(1-s, s, k ; \frac{x}{x-1}\right), & \text { if } 0<x<1 \\ \frac{\Gamma(k-s)}{\Gamma(s)} \frac{2^{2 s-2} \Gamma\left(s-\frac{1}{2}\right)}{\sqrt{\pi} \Gamma(k+s-1)}, & \text { if } x=1 \\ \frac{\Gamma(k-s)}{\Gamma(k) \Gamma(s)} x^{\frac{-k+1}{2}}(x-1)^{s-1} F\left(1-s, s, k ; \frac{1}{1-x}\right), & \text { if } x>1,\end{cases}
$$

where $F(\alpha, \beta, \gamma ; z)$ denotes the Gauss hypergeometric function defined for $|z|<1$ by the absolutely convergent series

$$
F(\alpha, \beta, \gamma ; z)=1+\frac{\alpha \cdot \beta}{\gamma \cdot 1} z+\frac{\alpha(\alpha+1) \beta(\beta+1)}{\gamma(\gamma+1) \cdot 1 \cdot 2} z^{2}+\cdots
$$

and for all values of $z$ by analytic continuation.

Combining these formulas we obtain: 
Proposition 3.6. Define $\gamma(s)=\frac{\Gamma(s) \Gamma(k)}{\Gamma(k-s)} \frac{\zeta(2 s)}{(2 \pi)^{2 s}}$. Then we have

$$
\gamma(s) L\left(s, P_{m} \otimes g\right)=\sum_{n=1}^{\infty} b(n) \gamma(m, n ; s)
$$

where

$$
\gamma(m, n ; s)= \begin{cases}\left(\frac{n}{m}\right)^{\frac{k-1}{2}} \sigma_{1-2 s}^{*}(m-n) F\left(1-s, s, k ; \frac{n}{n-m}\right) & \text { if } n<m \\ \gamma(s) m^{-s}+\gamma(1-s) m^{s-1} & \text { if } n=m \\ \left(\frac{m}{n}\right)^{\frac{k-1}{2}} \sigma_{1-2 s}^{*}(n-m) F\left(1-s, s, k ; \frac{n}{m-n}\right) & \text { if } n>m\end{cases}
$$

and $\sigma_{\nu}^{*}(n)=n^{-\frac{\nu+1}{2}} \sum_{d \mid n} d^{\nu}$ for positive integers $n$ and complex $\nu$.

Note that Proposition (3.6) (for the group $\Gamma=\Gamma_{0}(1)$ ) is also easily obtained by the standard Rankin-Selberg method. By unfolding the Poincaré series $P_{m}$ instead of the Eisenstein series $E(z, s)$ we obtain

$$
\begin{aligned}
\int_{\Gamma \backslash \mathfrak{h}} y^{k} \overline{P_{m}(z)} g(z) E(z, s) \frac{d x d y}{y^{2}} & =m^{\frac{k-1}{2}} \int_{\Gamma \backslash \mathfrak{h}} y^{k} \overline{e^{2 \pi i m z}} g(z) E(z, s) \frac{d x d y}{y^{2}} \\
& =\sum_{n=1}^{\infty}(m n)^{\frac{k-1}{2}} b(n) \int_{0}^{\infty} y^{k-2} e^{-2 \pi(m+n) y} e_{m-n}(s, y) d y
\end{aligned}
$$

where $e_{r}(s, y)$ denotes the coefficient of $e^{2 \pi i r x}$ in the Fourier expansion of $E(z, s)$. The formula for these Fourier coefficients is well-known: $e_{r}(s, y)$ is the product of $\sigma_{1-2 s}^{*}(|r|)$ and a simple analytic function of $|r| y$ for $r \neq 0$, and a linear combination of $\zeta(2 s) y^{s}$ and $\zeta(2 s-1) y^{1-s}$ for $r=0$. Substituting this into the above unfolding identity immediately gives (3.6).

REMARKs. The expression for $\gamma(s) L\left(s, P_{m} \otimes g\right)$ (on the right hand side in Proposition (3.6)) is absolutely convergent for all $s$ and each term is invariant under $s \rightarrow 1-s$ except the first two, which are interchanged, so one immediately deduces the meromorphic continuation and functional equation. It follows that $L\left(s, P_{m} \otimes g\right)$ is holomorphic everywhere except for a simple pole at $s=1$ with residue proportional to $b(m)$. The classical results of Rankin [R] and Selberg [S1] are immediately recovered.

The classical Rankin-Selberg proof is simpler than our new method if $f$ and $g$ are the same level and if $g$ is a cusp form. Otherwise, unfolding $P_{m}$ will force one to take the trace of $g E$ first, and it will be necessary to truncate $\operatorname{Tr}(g E)$ in order to make the integral convergent. This is more complicated than our new method given here and is very close to the original Gross-Zagier method. Our method was discovered by trying to simplify the proof of the Gross-Zagier formula. In that case $g$ is a theta function (not a cusp form) of different level than $f$ and our method avoids taking the trace and doing a holomorphic projection.

The formula (3.6) may yield new applications. For example, the rapid convergence of this formula, and the fact that that it is true also for $s$ outside the region of convergence of the original Dirichlet series $L(s, f \otimes g)$, might make it suitable for certain theoretical or computational applications. Also, the fact that $F(1-s, s, k ; x)$ becomes a polynomial for integral values of $s$ might be useful for obtaining new results, or new proofs of known results, about special values of $L(s, f \otimes g)$ at such arguments. It would also be of interest to see if our new method can be used to obtain higher convolutions of Rankin-Selberg type. 
4. Functional Equation for $L_{g}\left(s, \frac{r}{c}\right)$. In this section we derive the functional equation for

$$
L_{g}\left(s, \frac{r}{c}\right)=\sum_{n=1}^{\infty} b(n) e^{\frac{2 \pi i n r}{c}} n^{-s}
$$

where $g \in M_{\ell}\left(\Gamma_{0}(D), \epsilon\right)$. Here, we assume that $\ell$ is a fixed positive integer and that $\epsilon$ is a Dirichlet character of $(\mathbb{Z} / D \mathbb{Z})^{\times}$.

Let $\gamma=\left(\begin{array}{ll}a & b \\ c & d\end{array}\right)$ be a matrix with real entries and positive determinant. Given, $F(z)$ a holomorphic function on the upper half plane, define

$$
\left.F\right|_{\gamma}(z)=(a d-b c)^{\frac{\ell}{2}}(c z+d)^{-\ell} F(z)
$$

which satisfies $\left.\left.F\right|_{\gamma}\right|_{\gamma^{\prime}}=\left.F\right|_{\gamma \gamma^{\prime}}$. Set

Assume now that $D$ is square free. Let $\epsilon=\prod_{p \mid D} \epsilon_{p}$ be the decomposition of $\epsilon$.

$$
\delta=\frac{D}{(c, D)}, \quad \delta^{\prime}=(c, D)
$$

Since $\left(\delta, \delta^{\prime}\right)=1$ it follows that there exist $x, y \in \mathbb{Z}$ such that $x \delta-y \delta^{\prime}=1$. Define a matrix $w_{\delta}$ by the formula

$$
w_{\delta}=\left(\begin{array}{ll}
x & y \\
\delta^{\prime} & \delta
\end{array}\right)\left(\begin{array}{ll}
\delta & 0 \\
0 & 1
\end{array}\right)
$$

Then $w_{\delta}$ normalizes the subgroup $\Gamma_{0}(D)$.

Define

$$
g^{\delta}(z)=\left.g\right|_{w_{\delta}}(z)
$$

Then $g^{\delta}$ belongs to $M_{\ell}\left(\Gamma_{0}(D), \epsilon^{\delta}\right)$ where

$$
\epsilon^{\delta}=\epsilon_{\delta}^{-1} \cdot \epsilon_{\delta^{\prime}}=\prod_{p \mid \delta} \epsilon_{p}^{-1} \cdot \prod_{p \mid \delta^{\prime}} \epsilon_{p} .
$$

Proposition 4.2. The function $L_{g}\left(s, \frac{a}{c}\right)$ has a meromorphic continuation to the entire complex $s$-plane with simple poles at $s=\frac{\ell+1}{2}, \frac{1-\ell}{2}$ (with residue $-b(0)$ at $s=$ $\frac{1-\ell}{2}$ ) and satisfies the functional equation

$$
L_{g}\left(s, \frac{a}{c}\right)=\epsilon\left(\frac{a}{c}\right)\left(\frac{\delta c^{2}}{4 \pi^{2}}\right)^{\frac{1}{2}-s} \frac{\Gamma\left(\frac{\ell+1}{2}-s\right)}{\Gamma\left(\frac{\ell-1}{2}+s\right)} L_{g^{\delta}}\left(1-s,-\frac{\bar{a} \bar{\delta}}{c}\right)
$$

where

$$
\epsilon\left(\frac{a}{c}\right)=i^{-\ell} \epsilon_{\delta}\left(\frac{c}{\delta^{\prime}}\right) \epsilon_{\delta^{\prime}}^{-1}(a \delta)
$$

and $\bar{a}$ is the inverse of $a(\bmod c)$. Write

Proof. Since $(c, \delta)=1$, there exists a matrix $\gamma=\left(\begin{array}{ll}a & b \\ c & d\end{array}\right) \in \mathrm{SL}(\mathbb{Z})$ with $\delta \mid d$.

$$
\gamma^{\prime}=\gamma\left(\begin{array}{cc}
x & y \\
\delta^{\prime} & \delta
\end{array}\right)^{-1}=\left(\begin{array}{ll}
a \delta-b \delta^{\prime} & -a y+b x \\
c \delta-d \delta^{\prime} & -c y+d x
\end{array}\right)
$$


Then $\gamma^{\prime} \in \Gamma_{0}(D)$ since $\delta\left|d, \delta^{\prime}\right| c$, and $\delta \delta^{\prime}=D$. Since

$$
\gamma=\gamma^{\prime} w_{\delta}\left(\begin{array}{cc}
\delta^{-1} & 0 \\
0 & 1
\end{array}\right)
$$

we obtain

$$
\left.g\right|_{\gamma}(z)=\epsilon(-c y+d x) \delta^{-\frac{\ell}{2}} g^{\delta}\left(\frac{z}{\delta}\right)
$$

Here

$$
\epsilon(-c y+d x)=\epsilon_{\delta}(-c y) \epsilon_{\delta^{\prime}}(d x)=\epsilon_{\delta}\left(\frac{c}{\delta^{\prime}}\right) \epsilon_{\delta^{\prime}}^{-1}(a \delta)
$$

Write

$$
\frac{a z+b}{c z+d}=\frac{a}{c}-\frac{1}{c(c z+d)}
$$

and make the substitution $z \rightarrow-\frac{1}{c^{2} z}-\frac{d}{c}$. We have

$$
g\left(\frac{a}{c}+z\right)(c z)^{\ell}=\epsilon_{\delta}\left(\frac{c}{\delta^{\prime}}\right) \epsilon_{\delta^{\prime}}^{-\ell}(a \delta) \delta^{-\frac{\ell}{2}} g^{\delta}\left(-\frac{1}{\delta c^{2} z}+\frac{a^{\prime}}{c}\right)
$$

where $a^{\prime}=-d / \delta$.

Let $L_{g}^{*}\left(s, \frac{a}{c}\right)$ denote the Mellin transformation

$$
L_{g}^{*}\left(s, \frac{a}{c}\right)=\int_{0}^{\infty}\left[g\left(\frac{a}{c}+i y\right)-b(0)\right] y^{\frac{\ell-1}{2}+s} \frac{d y}{y}
$$

where $b(0)=0$ if $g$ is a cusp form. Then we have

$$
L_{g}^{*}\left(s, \frac{a}{c}\right)=\frac{\Gamma\left(\frac{\ell-1}{2}+s\right)}{(2 \pi)^{\frac{\ell-1}{2}+s}} L_{g}\left(s, \frac{a}{c}\right) .
$$

Now

$$
L_{g}^{*}\left(s, \frac{a}{c}\right)=\left(\int_{0}^{\frac{1}{c \sqrt{\delta}}}+\int_{\frac{1}{c \sqrt{\delta}}}^{\infty}\right)\left[g\left(\frac{a}{c}+i y\right)-b(0)\right] y^{\frac{\ell-1}{2}+s} \frac{d y}{y} .
$$

On the other hand, from the functional equation of $g$ and $g^{\delta}$, we have

$$
\begin{aligned}
& \int_{0}^{\frac{1}{c \sqrt{\delta}}}\left[g\left(\frac{a}{c}+i y\right)-b(0)\right] y^{\frac{\ell-1}{2}+s} \frac{d y}{y} \\
&=A \int_{0}^{\frac{1}{c \sqrt{\delta}}}\left[g^{\delta}\left(-\frac{1}{\delta c^{2} i y}+\frac{a^{\prime}}{c}\right)-b(0)\right](c i y)^{-\ell} y^{\frac{\ell-1}{2}+s} \frac{d y}{y} \\
&+A b(0)(c i)^{-\ell}(c \sqrt{\delta})^{-s+\frac{\ell+1}{2}} \cdot \frac{1}{s-\frac{\ell+1}{2}}-b(0)(c \sqrt{\delta})^{-s-\frac{\ell-1}{2}} \cdot \frac{1}{s+\frac{\ell-1}{2}}
\end{aligned}
$$

where

$$
A=\epsilon_{\delta}\left(\frac{c}{\delta^{\prime}}\right) \epsilon_{\delta^{\prime}}^{-1}(a \delta) \delta^{-\frac{\ell}{2}}
$$


If we make the substitution $y \rightarrow \frac{1}{c^{2} \delta y}$, we obtain

$$
\begin{aligned}
\int_{0}^{\frac{1}{c \sqrt{\delta}}}\left[g\left(\frac{a}{c}+i y\right)-b(0)\right] y^{\frac{\ell-1}{2}+s} \frac{d y}{y} \\
=A \int_{\frac{1}{c \sqrt{\delta}}}^{\infty}\left[g^{\delta}\left(\frac{a^{\prime}}{c}+i y\right)-b(0)\right](c i)^{-\ell}\left(c^{2} \delta y\right)^{\frac{\ell+1}{2}-s} \frac{d y}{y} \\
+A b(0)(c i)^{-\ell}(c \sqrt{\delta})^{-s+\frac{\ell+1}{2}} \cdot \frac{1}{s-\frac{\ell+1}{2}}-b(0)(c \sqrt{\delta})^{-s-\frac{\ell-1}{2}} \cdot \frac{1}{s+\frac{\ell-1}{2}} \\
\epsilon_{\delta}\left(\frac{c}{\delta^{\prime}}\right) \epsilon_{\delta^{\prime}}^{-1}(a \delta) \delta^{-\frac{\ell}{2}} \int_{0}^{\infty} g^{\delta}\left(-\frac{1}{\delta c^{2} i y}+\frac{a^{\prime}}{c}\right)(c i y)^{-\ell} y^{\frac{\ell-1}{2}+s} \frac{d y}{y}
\end{aligned}
$$

The functional equation

$$
L_{g}^{*}\left(s, \frac{a}{c}\right)=i^{-\ell} \epsilon_{\delta}\left(\frac{c}{\delta^{\prime}}\right) \epsilon_{\delta^{\prime}}^{-1}(a \delta)\left(\delta c^{2}\right)^{1 / 2-s} L_{g^{\delta}}^{*}\left(1-s, \frac{a^{\prime}}{c}\right)
$$

and Proposition (4.2) immediately follow.

5. Generalized Ramanujan Sums. In this section we fix a decomposition $D=\delta \cdot \delta^{\prime}$ of the square-free integer $D$. For any integer $A$ we decompose

$$
A=A_{1} A_{2}
$$

so that $A_{1}$ is positive with prime factors dividing $\delta^{\prime}$ and $A_{2}$ is prime to $\delta^{\prime}$.

DEFINITION 5.2. We define

$$
G(\delta)=\prod_{p \mid \delta^{\prime}} \epsilon_{p}^{-1}\left(\frac{\delta^{\prime}}{p}\right) \sum_{r \in(\mathbb{Z} / p \mathbb{Z})^{\times}} \epsilon_{p}^{-1}(r) e^{\frac{2 \pi i r}{p}} .
$$

Let $c$ be a positive integer and $B \in \mathbb{Z}$. The sum

$$
\sum_{r \in(\mathbb{Z} / c \mathbb{Z})^{\times}} \epsilon_{\delta^{\prime}}(r) e^{\frac{2 \pi i B r}{c}}
$$

is a generalized Ramanujan sum. We evaluate it in the next lemma using the notation $e^{x}=\exp (x)$.

Lemma 5.3. Let $c, B$ positive integers with $c>0,(c, D)=\delta^{\prime}$, and $B \neq 0$. Set $c=c_{1} c_{2}, B=B_{1} B_{2}$ as in (5.1). Then the sum

$$
\sum_{r \in(\mathbb{Z} / c \mathbb{Z})^{\times}} \epsilon_{\delta^{\prime}}(r) \exp \left(\frac{2 \pi i B}{c} r\right)
$$

is equal to (with $G(\delta)$ given in Definition 5.2)

$$
G(\delta) B_{1} \epsilon_{\delta^{\prime}}\left(\frac{B_{2}}{c_{2}}\right) \sum_{d \mid\left(c_{2}, B_{2}\right)} \mu\left(\frac{c_{2}}{d}\right) d
$$

if $c_{1}=B_{1} \delta^{\prime}$; otherwise it is zero. 
Proof. Let $c=\prod_{i=1}^{s} p_{i}^{n_{i}}$ be the prime decomposition of $c$ with $n_{i}>0$. Then every $r \in(\mathbb{Z} / c \mathbb{Z})^{\times}$can be uniquely written as $\sum_{i} r_{i}\left(c / p_{i}^{n_{i}}\right)$ with $r_{i} \in\left(\mathbb{Z} / p_{i}^{n_{i}} \mathbb{Z}\right)^{\times}$. Since

$$
\epsilon_{\delta^{\prime}}\left(\sum r_{i} c / p_{i}^{n_{i}}\right)=\prod_{p_{i} \mid \delta^{\prime}} \epsilon_{p_{i}}\left(\left(c / p_{i}^{n_{i}}\right) r_{i}\right)
$$

one has

$$
\begin{aligned}
\sum_{r \in(\mathbb{Z} / c \mathbb{Z})^{\times}} \epsilon_{\delta^{\prime}}(r)^{-1} \exp \left(\frac{2 \pi i B}{c} r\right)= & \prod_{p_{i} \mid \delta^{\prime}} \sum_{r \in\left(\mathbb{Z} / p_{i}^{n_{i}} \mathbb{Z}\right)^{\times}} \epsilon_{p_{i}}^{-1}\left(\left(c / p_{i}^{n_{i}}\right) r\right) \exp \left(\frac{2 \pi i B}{p_{i}^{n_{i}} r}\right) . \\
& \cdot \prod_{p_{i} \mid \delta^{\prime}} \sum_{r \in\left(\mathbb{Z} / p_{i}^{n_{i}} \mathbb{Z}\right)^{\times}} \exp \left(\frac{2 \pi i B}{p_{i}^{n_{i}}} r\right) .
\end{aligned}
$$

Let's evaluate the two products separately. If $p_{i} \mid \delta^{\prime}$, then every element in $\mathbb{Z} / p_{i}^{n_{i}} \mathbb{Z}$ can be uniquely written as $r+t p_{i}$ with $r \in\left(\mathbb{Z} / p_{i} \mathbb{Z}\right)^{\times}$and $t \in \mathbb{Z} / p_{i}^{n_{i}-1} \mathbb{Z}$. It follows that

$$
\begin{aligned}
& \sum_{r \in\left(\mathbb{Z} / p_{i}^{n_{i}} \mathbb{Z}\right)^{\times}} \epsilon_{p_{i}}^{-1}\left(\left(c / p_{i}^{n_{i}}\right) r\right) \exp \left(\frac{2 \pi i B}{p_{i}^{n_{i}}} r\right) \\
= & \sum_{r \in\left(\mathbb{Z} / p_{i} F\right)^{\times}} \epsilon_{p_{i}}^{-1}\left(\left(c / p_{i}^{n_{i}}\right) r\right) \exp \left(\frac{2 \pi i B}{p_{i}^{n_{i}}} r\right) \sum_{t \in \mathbb{Z} / p_{i}^{n_{i}-1} \mathbb{Z}} \exp \left(\frac{2 \pi i B}{p_{i}^{n_{i}-1}} t\right) .
\end{aligned}
$$

If $\operatorname{ord}_{p_{i}}(B)<n_{i}-1$, the last sum is zero; otherwise it is

$$
p_{i}^{n_{i}-1} \sum_{r \in\left(\mathbb{Z} / p_{i} \mathbb{Z}\right)^{\times}} \epsilon_{p_{i}}^{-1}\left(\left(c / p_{i}^{n_{i}}\right) r\right) \exp \left(\frac{2 \pi i B}{p_{i}^{n_{i}}} r\right) .
$$

Again this sum is 0 if $\operatorname{ord}_{p_{i}}(B) \geq n_{i}$. Otherwise, replace $r$ by $r\left(B / p_{i}^{n_{i}-1}\right)^{-1}\left(\bmod p_{i}\right)$ to obtain

$$
p_{i}^{n_{i}-1} \epsilon_{p_{i}}\left(B p_{i} / c\right) \sum_{r \in\left(\mathbb{Z} / p_{i} \mathbb{Z}\right)^{\times}} \epsilon_{p_{i}}^{-1}(r) \exp \left(\frac{2 \pi i}{p_{i}} r\right) .
$$

It follows that

$$
\prod_{p_{i} \mid \delta^{\prime}} \sum_{r \in\left(\mathbb{Z} / p_{i}^{n_{i}} \mathbb{Z}\right)^{\times}} \epsilon_{p_{i}}^{-1}\left(\left(c / p_{i}^{n_{i}}\right) r\right) \exp \left(\frac{2 \pi i B}{p_{i}^{n_{i}}} r\right)
$$

is nonzero only if $B_{1} \delta^{\prime}=c_{1}$; in this case, it is equal to

$$
B_{1} \epsilon_{\delta^{\prime}}\left(B_{2} / c_{2}\right) G(\delta)
$$

Now, we assume that $p_{i} \not \delta^{\prime}$, then

$$
\begin{aligned}
\sum_{r \in\left(\mathbb{Z} / p_{i}^{n_{i}} \mathbb{Z}\right)^{\times}} \exp \left(\frac{2 \pi i B}{p_{i}^{n_{i}} r}\right) & =\sum_{r \in \mathbb{Z} / p_{i}^{n_{i}} \mathbb{Z}} \exp \left(\frac{2 \pi i B}{p_{i}^{n_{i}}} r\right)-\sum_{r \in \mathbb{Z} / p_{i}^{n_{i}-1} \mathbb{Z}} \exp \left(\frac{2 \pi i B}{p_{i}^{n_{i}}} r p\right) \\
& =\sum_{d \mid\left(B, p_{i}^{n_{i}}\right)} \mu\left(\frac{p_{i}^{n_{i}}}{d}\right) d .
\end{aligned}
$$

It follows that

$$
\prod_{p_{i} \mid \delta^{\prime}} \sum_{r \in\left(\mathbb{Z} / p_{i}^{n_{i}} \mathbb{Z}\right)^{\times}} \exp \left(\frac{2 \pi i B}{p_{i}^{n_{i}}} r\right)=\sum_{d \mid\left(c_{2}, B\right)} \mu\left(\frac{c}{d}\right) d .
$$


This completes the proof of Lemma 5.3.

6. The Holomorphic Kernel $\Phi_{s, g}$. We recall formulas (3.4), (3.5) which we now relabel as $(6.1), 6.2)$.

$$
L\left(s, P_{m} \otimes g\right)=b(m) m^{-s}+2 \pi i^{k} T_{m}(s)
$$

$T_{m}(s)=\sum_{\substack{c=1 \\ N \mid c}}^{\infty} \sum_{n=1}^{\infty} \frac{S(m, n: c)}{c} \frac{b(n)}{n^{s}} \cdot \frac{1}{2 \pi i} \int_{\epsilon-\frac{k-1}{2}-i \infty}^{\epsilon-\frac{k-1}{2}+i \infty} \frac{\Gamma\left(\frac{k-1}{2}+w\right)}{\Gamma\left(\frac{k+1}{2}-w\right)}\left(\frac{2 \pi \sqrt{m n}}{c}\right)^{-2 w} d w$

$$
=\sum_{\substack{c=1 \\ N \mid c}}^{\infty} \sum_{r \in(\mathbb{Z} / c \mathbb{Z})^{\times}} e^{\frac{2 \pi i m . r}{c}} \cdot \frac{1}{2 \pi i} \int_{\epsilon-\frac{k-1}{2}-i \infty}^{\epsilon-\frac{k-1}{2}+i \infty} \frac{\Gamma\left(\frac{k-1}{2}+w\right)}{\Gamma\left(\frac{k+1}{2}-w\right)} \frac{(2 \pi \sqrt{m})^{-2 w}}{c^{1-2 w}} L_{g}\left(s+w, \frac{\bar{r}}{c}\right) d w
$$

and

$$
L_{g}\left(s, \frac{r}{c}\right)=\sum_{n=1}^{\infty} b(n) e^{\frac{2 \pi i n n}{c}} n^{-s}
$$

with $r \bar{r} \equiv 1(\bmod c)$. Since $L_{g}\left(s, \frac{r}{c}\right)$ is holomorphic in $s$, formula (6.2) holds for all $s$.

In (6.2) we will apply the functional equation given in Proposition 4.2. The Mellin-Barnes integral (for $x>0, s \in \mathbb{C}, \operatorname{Re}(s)>1$ )

$$
I_{s}(x)=\frac{1}{2 \pi i} \int_{\epsilon-\frac{k-1}{2}-i \infty}^{\epsilon-\frac{k-1}{2}+i \infty} \frac{\Gamma\left(\frac{k-1}{2}+w\right) \Gamma\left(\frac{\ell+1}{2}-s-w\right)}{\Gamma\left(\frac{k+1}{2}-w\right) \Gamma\left(\frac{\ell-1}{2}+s+w\right)} x^{-w} d w
$$

naturally appears. This integral is evaluated in Proposition 8.3. Further, the Kloosterman sums then turn into generalized Ramanujan sums (here $B \in \mathbb{Z}, s \in \mathbb{C}$ with $\operatorname{Re}(s)>1)$

$$
S^{\delta}(s, B)=\sum_{\substack{c=1 \\ N \mid c \\(c, D)=\delta^{\prime}}}^{\infty} \frac{\epsilon_{\delta}\left(c / \delta^{\prime}\right)}{c^{2 s}} \sum_{r \in(\mathbb{Z} / c \mathbb{Z})^{\times}} \epsilon_{\delta^{\prime}}^{-1}(r) \exp \left(\frac{2 \pi i B}{c} r\right) .
$$

These sums are evaluated by Lemma 5.3 in Proposition 7.1. This is the key idea for obtaining the final formula for the holomorphic kernel as given in Theorem 6.5 which is the main Theorem of this paper.

THEOREM 6.5. Fix positive integers $k, \ell, N, D$ and

$$
g(z)=b(0)+\sum_{n=1}^{\infty} b(n) n^{\frac{\ell-1}{2}} e^{2 \pi i n z}
$$

in $M_{\ell}\left(\Gamma_{0}(D), \epsilon\right)$. Assume that $D$ is square free, $\epsilon$ a Dirichlet character $(\bmod D)$, and $s \in \mathbb{C}$. Then we have: 
(a) The kernel function $\Phi_{s, g}(z)$ defined in (3.1), (3.2) has the Fourier expansion

$$
\Phi_{s, g}(z)=\frac{(4 \pi)^{k-1}}{(k-2) !} \sum_{m=1}^{\infty} L\left(s, P_{m} \otimes g\right) e^{2 \pi i m z}
$$

(b) The function $L\left(s, P_{m} \otimes g\right)$ is given by

$$
L\left(s, P_{m} \otimes g\right)=b(m) m^{-s}+2 \pi i^{k} \sum_{\delta \mid D} T_{m}^{\delta}(s)
$$

with

$$
T_{m}^{\delta}(s)=i^{-\ell}\left(\frac{\delta}{4 \pi^{2}}\right)^{\frac{1}{2}-s} \epsilon_{\frac{D}{\delta}}^{-2}(\delta) \sum_{n=1}^{\infty} \frac{b^{\delta}(n)}{n^{1-s}} S^{\delta}(s, m \delta-n) I_{s}\left(\frac{\delta m}{n}\right)
$$

where $b^{\delta}(n)$ are fourier coefficients of $g^{\delta}$ defined in $\S 3, I_{s}(x)$ is the Mellin-Barnes integral (6.3), and $S^{\delta}(s, B)$ is the generalized Ramanujan sum 6.4.

Proof. It follows from the functional equation given in Proposition 4.2 that

$$
\begin{aligned}
\frac{(2 \pi \sqrt{m})^{-2 w}}{c^{1-2 w}} & L_{g}\left(s+w, \frac{\bar{r}}{c}\right)= \\
& \epsilon\left(\frac{a}{c}\right) \frac{(\delta m)^{-w}}{c^{2 s}}\left(\frac{\delta}{4 \pi^{2}}\right)^{\frac{1}{2}-s} \frac{L\left(\frac{\ell+1}{2}-s-w\right)}{L\left(\frac{\ell-1}{2}+s+w\right)} \cdot L_{g^{\delta}}\left(1-s-w,-\frac{\bar{a} \bar{\delta}}{c}\right) .
\end{aligned}
$$

If we use this identity in equation (6.2) and recall that

$$
g^{\delta}(z)=\sum_{n \geq 0} b^{\delta}(n) n^{\frac{\ell-1}{2}} e^{2 \pi i n z}
$$

and

$$
L_{g^{\delta}}\left(s, \frac{r}{c}\right)=\sum_{n=1}^{\infty} b^{\delta}(n) e^{\frac{2 \pi i n r}{c}} n^{-s}
$$

it follows that

$$
T_{m}(s)=\sum_{\delta \mid D} T_{m}^{\delta}(s)
$$

where

$$
T_{m}^{\delta}(s)=i^{-\ell}\left(\frac{\delta}{4 \pi^{2}}\right)^{\frac{1}{2}-s} \epsilon_{\delta^{\prime}}^{-2}(\delta) \sum_{n=1}^{\infty} \frac{b^{\delta}(n)}{n^{1-s}} S^{\delta}(s, m \delta-n) I_{s}\left(\frac{\delta m}{n}\right)
$$

7. Evaluation of $S^{\delta}(s, B)$. As before, we work with a fixed decomposition $D=\delta \cdot \delta^{\prime}$ of the square-free integer $D$ and $\epsilon$ is a Dirichlet character $(\bmod D)$. Recall the definition of $\epsilon^{\delta}$ given in (4.1):

$$
\epsilon^{\delta}=\epsilon_{\delta}^{-1} \cdot \epsilon_{\delta^{\prime}}=\prod_{p \mid \delta} \epsilon_{p}^{-1} \cdot \prod_{p \mid \delta^{\prime}} \epsilon_{p}
$$


For any given number $e$ prime to $D$, let $L_{e}^{\delta}(s)$ denote the Dirichlet L-function

$$
L_{e}^{\delta}(s)=\sum_{(n, D e)=1} \frac{\epsilon^{\delta}\left(\frac{1}{n}\right)}{n^{s}} .
$$

When $e=1$ we simply denote it by $L^{\delta}(s)$.

Proposition 7.1. Let $B$ be an integer with decomposition $B=B_{1} B_{2}$ as in (5.1). Let $N=N_{1} N_{2}$ as in (5.1). Define

$$
S_{e}^{\delta}(s, B)= \begin{cases}\frac{G(\delta) \epsilon_{\delta}\left(B_{1}\right) \epsilon_{\delta^{\prime}}\left(B_{2}\right)}{\left(\delta^{\prime}\right)^{2 s} B_{1}^{2 s-1}} \sum_{\substack{d \mid \frac{B_{2}}{e} \\(d, D)=1}} d^{1-2 s} \epsilon^{\delta}\left(\frac{1}{d}\right) . & B \neq 0, N \mid B_{1} \delta^{\prime} \\ L^{\delta}(2 s-1) & B=0, \delta=D,(N, D)=1 \\ 0 & \text { otherwise }\end{cases}
$$

Then

$$
S^{\delta}(s, B)=\frac{\epsilon^{\delta}\left(1 / N_{2}\right) N_{2}^{1-2 s}}{L_{N_{2}}^{\delta}(2 s)} \sum_{e \mid\left(B_{2}, N_{2}\right)} \mu\left(\frac{N_{2}}{e}\right) \frac{e}{N_{2}} S_{e}^{\delta}(s, B) .
$$

Proof. Assume that $B \neq 0$ first. By Lemma 5.3, if $S^{\delta}(s, B) \neq 0$ then there is a positive integer $c$ such that $N \mid c,(c, D)=\delta^{\prime}$, and $c_{1}=B_{1} \delta^{\prime}$. This implies that $N_{1} \mid B_{1} \delta^{\prime}$. Assuming this, Lemma 5.3 then gives

$$
S^{\delta}(s, B)=\sum_{\substack{N_{2} \mid c_{2} \\\left(c_{2}, D\right)=1}} \frac{\epsilon_{\delta}\left(B_{1} c_{2}\right)}{\left(B_{1} \delta^{\prime} c_{2}\right)^{2 s}} G(\delta) B_{1} \epsilon_{\delta^{\prime}}\left(B_{2} / c_{2}\right) \sum_{d \mid\left(c_{2}, B_{2}\right)} \mu\left(c_{2} / d\right) d
$$

Interchanging the summation, we obtain

$$
S^{\delta}(s, B)=\frac{G(\delta) \epsilon_{\delta}\left(B_{1}\right) \epsilon_{\delta^{\prime}}\left(B_{2}\right)}{\left(B_{1} \delta^{\prime}\right)^{2 s}} \sum_{d \mid B_{2}} d \sum_{\substack{N_{2}\left|c_{2} \\ d\right| c_{2} \\\left(c_{2}, D\right)=1}} \frac{\epsilon^{\delta}\left(1 / c_{2}\right) \mu\left(c_{2} / d\right)}{c_{2}^{2 s}}
$$

If $S^{\delta}(s, B) \neq 0$ then $\left(N_{2}, D\right)=1$. Assume this and let $e \mid N_{2}$ be a factor such that

$$
\left(\frac{c_{2}}{d}, N_{2}\right)=\frac{N_{2}}{e}
$$

Substituting $c_{2}$ by $d \frac{N_{2}}{e} \ell$, then $(e, \ell)=1$, and we obtain

$$
\begin{gathered}
S^{\delta}(s, B)=\frac{G(\delta) \epsilon_{\delta}\left(B_{1} \delta^{\prime}\right) \epsilon_{\delta^{\prime}}\left(B_{2}\right)}{\left(\delta^{\prime}\right)^{2 s} B_{1}^{2 s-1}} \sum_{\substack{d \mid B_{2} \\
(d, D)=1}} \sum_{\substack{e\left|N_{2} \\
e\right| d}} d \frac{\epsilon^{\delta}\left(1 /\left(d N_{2} e^{-1}\right)\right) \mu\left(N_{2} / e\right)}{\left(d N_{2} / e\right)^{2 s}} . \\
\cdot \sum_{\substack{(\ell, D)=1 \\
(\ell, e)=1 \\
\left(\ell, N_{2} / e\right)=1}} \frac{\epsilon^{\delta}(1 / \ell) \mu(\ell)}{\ell^{2 s}} .
\end{gathered}
$$

Interchange the sums over $e$ and $d$ and replace $d$ by $d \cdot e$. The Proposition follows in the case $B \neq 0$. The case $B=0$ can be treated similarly. 
8. Evaluation of $I_{s}$. Let

$$
\begin{aligned}
F(\alpha, \beta, \gamma ; z)=1+\frac{\alpha \cdot \beta}{\gamma \cdot 1} z+ & \frac{\alpha(\alpha+1)(\beta(\beta+1)}{\gamma(\gamma+1) \cdot 1 \cdot 2} z^{2} \\
& +\frac{\alpha(\alpha+1)(\alpha+2) \beta(\beta+1)(\beta+2)}{\gamma(\gamma+1)(\gamma+2) \cdot 1 \cdot 2 \cdot 3} z^{3}+\cdots
\end{aligned}
$$

denote the hypergeometric function. It is well known that the hypergeometric function $F$ satisfies the following identities:

$$
\begin{aligned}
& F(\alpha, \beta, \gamma ; z)=F(\beta, \alpha, \gamma ; z) \\
& F(\alpha, \beta, \gamma ; z)=(1-z)^{-\alpha} F\left(\alpha, \gamma-\beta, \gamma ; \frac{z}{z-1}\right)
\end{aligned}
$$

We use these identities to prove the following:

Proposition 8.3. Assume that $\operatorname{Re}(s)<\frac{k+\ell}{2}$. Then $I_{s}(x)$ is given by the following formulae:

$$
x^{\frac{k-1}{2}} \frac{\Gamma\left(\frac{\ell+k}{2}-s\right)}{\Gamma(k) \Gamma\left(\frac{\ell-k}{2}+s\right)}(1-x)^{\frac{\ell-k}{2}-1+s} F\left(\frac{k-\ell}{2}+s, \frac{k-\ell}{2}+1-s, k ; \frac{x}{x-1}\right),
$$

if $0<x<1$;

$$
\frac{\Gamma\left(\frac{\ell+k}{2}-s\right) \Gamma(2 s-1)}{\Gamma\left(\frac{k-\ell}{2}+s\right) \Gamma\left(\frac{\ell-k}{2}+s\right) \Gamma\left(\frac{\ell+k}{2}+s-1\right)}
$$

if $x=1$;

$$
\frac{\Gamma\left(\frac{\ell+k}{2}-s\right)}{\Gamma(\ell) \Gamma\left(\frac{k-\ell}{2}+s\right)} x^{\frac{-k+1}{2}}(x-1)^{\frac{k-\ell}{2}+s-1} F\left(\frac{\ell-k}{2}+1-s, \frac{\ell-k}{2}+s, k ; \frac{1}{1-x}\right),
$$

if $x>1$.

Proof. Recall formula (6.3)

$$
I_{s}(x)=\frac{1}{2 \pi i} \int_{\epsilon-\frac{k-1}{2}-i \infty}^{\epsilon-\frac{k-1}{2}+i \infty} \frac{\Gamma\left(\frac{k-1}{2}+w\right) \Gamma\left(\frac{\ell+1}{2}-s-w\right)}{\Gamma\left(\frac{k+1}{2}-w\right) \Gamma\left(\frac{\ell-1}{2}+s+w\right)} x^{-w} d w .
$$

For $0<x<1$, we compute the integral by shifting the line of integration to the left. The integrand has poles at $w=-\frac{k-1}{2}-n$ with $n=0,1,2, \ldots$ Consequently

$$
I_{s}(x)=\sum_{n=1}^{\infty} \frac{(-1)^{n}}{n !} \frac{\Gamma\left(\frac{\ell+k}{2}-s+n\right)}{\Gamma(k+n) \Gamma\left(\frac{\ell-k}{2}+s-n\right)} x^{\frac{k-1}{2}+n} .
$$

LEMMA 8.4. For $x>0$

$$
\sum_{n=0}^{\infty} \frac{(-1)^{n}}{n !} \frac{\Gamma(a+n)}{\Gamma(b+n) \Gamma(c-n)} x^{n}=\frac{\Gamma(a)}{\Gamma(b) \Gamma(c)} F(a, 1-c, b ; x) .
$$


Proof. By the properties $x \Gamma(x)=\Gamma(x+1), \Gamma(1)=1$ of the Gamma function, and the definition of the hypergeometric function $F$, we have

$$
\begin{aligned}
\sum_{n=0}^{\infty} \frac{(-1)^{n}}{n !} & \frac{\Gamma(a+n)}{\Gamma(b+n) \Gamma(c-n)} x^{n} \\
& =\frac{\Gamma(a)}{\Gamma(b) \Gamma(c)}\left(1-\frac{a \cdot(c-1)}{1 ! b} x+\frac{a(a+1)(c-1)(c-2)}{2 ! b(b+1)} x^{2}-\cdots\right) \\
& =\frac{\Gamma(a)}{\Gamma(b) \Gamma(c)} F(a, 1-c, b ; x),
\end{aligned}
$$

which concludes the proof of Lemma 8.4.

It follows from Lemma 8.4 that

$$
I_{s}(x)=x^{\frac{k-1}{2}} \frac{\Gamma\left(\frac{\ell+k}{2}-s\right)}{\Gamma(k) \Gamma\left(\frac{\ell-k}{2}+s\right)} F\left(\frac{k+\ell}{2}-s, \frac{k-\ell}{2}+1-s, k ; x\right) .
$$

We apply to this the first transformation (8.1) and then the functional equation (8.2) with $x \rightarrow x /(x-1)$. The first formula in Proposition 8.3 immediately follows.

For $x>1$, we must shift the line of integration to the right. The integrand has poles at $w=\frac{\ell+1}{2}-s+n$ with $n=0,1,2, \ldots$ We have

$$
I_{s}(x)=\sum_{n \geq 0} \frac{(-1)^{n}}{n !} \frac{\Gamma\left(\frac{k+\ell}{2}-s+n\right)}{\Gamma\left(\frac{k-\ell}{2}+s-n\right) \Gamma(\ell+n)} x^{-\frac{\ell+1}{2}+s-n} .
$$

Applying Lemma 8.4, we have

$$
I_{s}(x)=x^{-\frac{\ell+1}{2}+s} \frac{\Gamma\left(\frac{k+\ell}{2}-s\right)}{\Gamma\left(\frac{k-\ell}{2}+s\right) \Gamma(\ell)} F\left(\frac{k+\ell}{2}-s, \frac{\ell-k}{2}+1-s, \ell ; x^{-1}\right) .
$$

Again, the transformation (8.1) and the functional equation (8.2) $\frac{1}{x} \rightarrow \frac{1}{x} /\left(\frac{1}{x}-1\right)=$ $1 /(x-1)$ gives the formula in Proposition 8.3 in the case $x>1$.

In the remaining case when $x=1$, we require the following lemma.

LEMMA 8.5.

$$
F(a, b, c ; 1)=\frac{\Gamma(c) \Gamma(c-a-b)}{\Gamma(c-a) \Gamma(c-b)} .
$$

Proof. Using the identity

$$
\frac{\Gamma(x) \Gamma(y)}{\Gamma(x+y)}=\int_{0}^{1} t^{x-1}(1-t)^{y-1} d t
$$

and the Tayler expansion of $(1-t z)^{a}$ at $z=0$, we obtain:

$$
F(a, b, c ; z)=\frac{\Gamma(c)}{\Gamma(b) \Gamma(c-b)} \int_{0}^{1}(1-t z)^{-a} t^{b-1}(1-t)^{c-b-1} d t .
$$

This gives the formula in lemma 8.5 after setting $z=1$.

The formula for $I_{s}(1)$ in Proposition 8.3 follows by applying lemma 8.5 to the case of the first formula for $I_{s}(x)$ when $0<x<1$. This completes the proof of Proposition 8.3 . 
Proposition 8.6. Define

$$
\tilde{I}_{s}(x)=\frac{\Gamma\left(\frac{k-\ell}{2}+s\right)}{\Gamma\left(\frac{k+\ell}{2}-s\right)} I_{s}(x) .
$$

Then for $x \neq 1$ we have the functional equation

$$
\frac{\tilde{I}_{1-s}(x)}{|x-1|^{1-s}}=\operatorname{sgn}(x-1)^{k-\ell} \frac{\tilde{I}_{s}(x)}{|x-1|^{s}} .
$$

Proof. Assume first that $0<x<1$. It follows from Proposition 8.3 that

$$
\frac{\tilde{I}_{1-s}(x)}{|x-1|^{1-s}}=\frac{\Gamma\left(\frac{k-\ell}{2}-s\right)}{\Gamma(k) \Gamma\left(\frac{\ell-k}{2}+s\right)}(1-x)^{\frac{\ell-k}{2}-1} F\left(\frac{k-\ell}{2}+s, \frac{k-\ell}{2}+1-s, k ; \frac{x}{x-1}\right) \text {. }
$$

By property (8.1), the hypergeometric function $F$ above is invariant under the transformation $s \rightarrow 1-s$. Further, since $k \equiv \ell(\bmod 2)$, we may set $a=\frac{k-\ell}{2} \in \frac{1}{2} \mathbb{Z}$. Then we must have

$$
\frac{\Gamma(a+s)}{\Gamma(-a+s)}=(-1)^{2 a} \frac{\Gamma(a+1-s)}{\Gamma(-a+1-s)}
$$

since

$$
\begin{aligned}
\Gamma(a+s) \Gamma(1-a-s) & =\frac{\pi}{\sin (\pi(a+s))} \\
& =(-1)^{2 a} \frac{\pi}{\sin (\pi(-a+s))} \\
& =(-1)^{2 a} \Gamma(-a+s) \Gamma(1-(-a+s)) .
\end{aligned}
$$

The functional equation immediately follows. In the case $x>1$, the proof is even easier since the gamma factors cancel out.

9. The Holomorphic Kernel $\widetilde{\Phi}_{s, g}$ for Real Characters. In general, $\Phi_{s, g}$, does not have a simple functional equation. However, in the case $\epsilon$ is a real quadratic character, then we can replace $\Phi_{s, g}$ by a new function $\widetilde{\Phi}_{s, g}$ which has simpler Fourier coefficients.

Proposition 9.1. Fix positive integers $k, \ell, N, D$ and

$$
g(z)=b(0)+\sum_{n=1}^{\infty} b(n) n^{\frac{\ell-1}{2}} e^{2 \pi i n z}
$$

in $M_{\ell}\left(\Gamma_{0}(D), \epsilon\right)$ with $\epsilon$ a real quadratic Dirichlet character $(\bmod D)$. For $s \in \mathbb{C}$ define

$$
\widetilde{\Phi}_{s, g}(z)=\sum \tilde{\phi}_{s, g}(m) m^{\frac{k-1}{2}} e^{2 \pi i m z}
$$

with

$$
\tilde{\phi}_{s, g}(m)=\frac{b(m)}{m^{s}} \sum_{e \mid N_{2}} \mu\left(\frac{N_{2}}{e}\right) \frac{e}{N_{2}} \cdot \frac{\Gamma\left(\frac{k-\ell}{2}+s\right) L_{e}(2 s)}{\Gamma\left(\frac{k+\ell}{2}-s\right)(2 \pi)^{2 s} \epsilon(e) e^{1-2 s}}+2 \pi i^{k} T_{m, N_{2}}(s)
$$

where

$$
T_{m, N_{2}}(s)=\frac{\Gamma\left(\frac{k-\ell}{2}+s\right)}{\Gamma\left(\frac{k+\ell}{2}-s\right)} \sum_{\delta \mid D} \frac{\delta^{\frac{1}{2}-s}}{2 \pi i^{\ell}} \sum_{n=1}^{\infty} \frac{b^{\delta}(n)}{n^{1-s}} S_{e}^{\delta}(s, m \delta-n) I_{s}\left(\frac{\delta m}{n}\right)
$$


$S_{N_{2}}^{\delta}(s, m \delta-n)$ is given in Proposition 6.5, and $I_{s}\left(\frac{\delta m}{n}\right)$ is given in (6.3).

Then $\widetilde{\Phi}_{s, g}(z)$ is a cusp form of weight $k$ for $\Gamma_{0}(N)$. Further, for any newform $f$ of weight $k$ for $\Gamma_{0}(N)$, we have

$$
L(s, f \otimes g)=\frac{(4 \pi)^{k-1}}{(k-2) !} \frac{\Gamma\left(\frac{k+\ell}{2}-s\right)(2 \pi)^{2 s} \epsilon\left(N_{2}\right) N_{2}^{1-2 s}}{\Gamma\left(\frac{k-\ell}{2}+s\right) L_{N_{2}}(2 s)} \cdot\left\langle\widetilde{\Phi}_{s, g}, f>.\right.
$$

Proof. Since $\epsilon$ is real we have $\epsilon^{2}=1$ and $\epsilon^{\delta}=\epsilon$. By Proposition $6.5, S_{e}^{\delta}(s, B) \neq 0$ only if $N \mid B \delta^{\prime}$ and $(N, \delta)=1$. In the decomposition $N=N_{1} N_{2}$ (as in (5.1)) we may, therefore, assume that $N_{2}$ is maximal and prime to $D$.

For any factor $e$ of $N_{2}$ define

$$
T_{m, e}=\sum_{\delta \mid D} T_{m, e}^{\delta}
$$

with $T_{m, e}^{\delta}$ given by the formula

$$
T_{m, e}^{\delta}(s)=\frac{\delta^{\frac{1}{2}-s}}{2 \pi i^{\ell}} \sum_{n=1}^{\infty} \frac{b^{\delta}(n)}{n^{1-s}} S_{e}^{\delta}(s, m \delta-n) \tilde{I}_{s}\left(\frac{\delta m}{n}\right)
$$

where $\tilde{I}_{s}$ is defined by

$$
\tilde{I}_{s}(x)=\frac{\Gamma\left(\frac{k-\ell}{2}+s\right)}{\Gamma\left(\frac{k+\ell}{2}-s\right)} I_{s}(x) .
$$

Define $\tilde{\phi}_{s, g}(m)$ by the formula

$$
\tilde{\phi}_{s, g}(m)=b(m) m^{-s} B_{s}\left(N_{2}\right)+2 \pi i^{k} T_{m, N_{2}}(s)
$$

where

$$
B_{s}\left(N_{2}\right)=\sum_{e \mid N_{2}} \mu\left(\frac{N_{2}}{e}\right) \frac{e}{N_{2}} A_{s}(e)^{-1}
$$

and

$$
A_{s}(e)=\frac{\Gamma\left(\frac{l+k}{2}-s\right)(2 \pi)^{2 s}}{\Gamma\left(\frac{k-l}{2}+s\right)} \frac{\epsilon(e) e^{1-2 s}}{L_{e}(2 s)} .
$$

By definition, $T_{m, e}(s)$ depends only on $N_{1} e$ and $m$. It follows from (6.6), (6.7), and Proposition 6.5 that

$$
T_{m}(s)=A_{s}\left(N_{2}\right) \sum_{e \mid N_{2}} \mu\left(\frac{N_{2}}{e}\right) \frac{e}{N_{2}} T_{m, e}(s) .
$$

It further follows from (6.1) and (9.2) that

$$
\tilde{\phi}_{s, g}(m)=\sum_{e \mid N_{2}} \mu\left(\frac{N_{2}}{e}\right) \frac{e}{N_{2}} A_{s}(e) L\left(s, P_{m, e} \otimes g\right)
$$

where $P_{m, e}$ denotes the $m^{\text {th }}$ Poincaré series for $\Gamma_{0}\left(N_{1} e\right)$.

10. The Functional Equation of $\widetilde{\Phi}_{s, g}$ when $D=1$. 
Theorem 10.1. Fix positive integers $k, \ell, N$ with $k \equiv \ell(\bmod 2)$. Fix a modular form $g \in M_{\ell}\left(\Gamma_{0}(1)\right)$. Define $\widetilde{\Phi}_{s, g}$ as in Theorem 9.1 with the choice $D=1$. Then $\widetilde{\Phi}_{s, g}$ has the Fourier coefficients

$$
\begin{aligned}
\tilde{\phi}_{s, g}(m)= & i^{k-\ell} N^{1-s} \frac{\Gamma(1-s) \Gamma(s)}{(2 \pi)^{2 s} \Gamma\left(\frac{\ell-k}{2}+1-s\right) \Gamma\left(\frac{\ell+k}{2}-s\right)} \zeta(2 s) \\
& +i^{k-\ell} N^{1-s} \frac{\Gamma(1-s) \Gamma(s)}{(2 \pi)^{2-2 s} \Gamma\left(\frac{\ell+k}{2}+s\right) \Gamma\left(\frac{\ell+k}{2}-1+s\right)} \zeta(2-2 s) \\
& +i^{k-\ell} N^{1-s} \sum_{\substack{n \geq 1, n \neq m D \\
n \equiv m(\bmod N)}} b(n) \sum_{\left|\frac{m-n}{N}\right|=d_{1} \cdot d_{2}} \frac{1}{d_{1}^{s} d_{2}^{1-s}}\left|\frac{m}{n}-1\right|^{1-s} \tilde{I}_{s}\left(\frac{m}{n}\right)
\end{aligned}
$$

and we have the functional equation

$$
\widetilde{\Phi}_{1-s, g}=N^{1-2 s} \widetilde{\Phi}_{s, g} .
$$

Proof. The formula follows from Proposition 9.1 by taking $D=1$ and $N_{2}=1$. The functional equation follows from the functional equation of $\zeta(s)$ and Proposition 8.6 .

11. The Functional Equation of $\widetilde{\Phi}_{s, g}$ when $g$ is a Theta Function. We now assume that $N$ is prime to $D$ and $\epsilon(-1)=-1$. Extend $\epsilon$ to a character on $\mathbb{A}^{\times} / \mathbb{Q}^{\times}$. Assume that

$$
g(z)=b(0)+\sum_{n=1}^{\infty} b(n) n^{\frac{\ell-1}{2}} e^{2 \pi i n z}
$$

transforms like a theta function attached to an imaginary quadratic field $\mathbb{Q}(\sqrt{-D})$ : In this case the Fourier coefficients $b(n)$ (with $n>0$ ) satisfy the following properties.

$$
\text { For any } \delta \mid D, b(\delta)= \pm 1
$$

$$
b(n) \neq 0 \text { only if } \epsilon(n)=1 \text {. }
$$

For $\delta \mid d, b(n \delta)=b(n) b(\delta)$.

$$
\text { For } \delta \mid D \text {, let } \kappa(\delta)=\sqrt{\epsilon_{\delta}(-1)} \text {. Then } b^{\delta}(n)=\kappa(\delta)^{-1} b(n \delta) \epsilon_{\delta}(n) \text {. }
$$

TheOREM 11.5. The function $\widetilde{\Phi}_{s, g}$ defined in $\S 9$ has Fourier coefficients given by

$$
\begin{aligned}
\tilde{\phi}_{s, g}(m)= & i^{k-\ell} L(2 s, \epsilon) \frac{D^{s} N^{2 s-1}}{(2 \pi)^{s}} \frac{\Gamma\left(\frac{1}{2}-s\right) \Gamma\left(\frac{1}{2}+s\right)}{\left(\frac{\ell-k}{2}+1-s\right) \Gamma\left(\frac{\ell+k}{2}-s\right)} \frac{b^{D}(m)}{m^{s}} \\
& +i^{k-\ell} L(2-2 s, \epsilon) \frac{D^{2-3 s}}{(2 \pi)^{2-2 s}} \frac{\Gamma\left(s-\frac{1}{2}\right) \Gamma\left(\frac{3}{2}-s\right)}{\Gamma\left(\frac{\ell-k}{2}+s\right) \Gamma\left(\frac{\ell+k}{2}-1+s\right)} \frac{b^{D}(m)}{m^{1-s}} \\
& +i^{k-\ell} D^{1 / 2-s} b(D) \kappa(D)^{-1} \sum_{\substack{n \geq 1, n \neq m D \\
m D \equiv n(\bmod N)}} \frac{b(n)}{n^{1-s}} J_{s}\left(\frac{m D-n}{N}\right) \tilde{I}_{s}\left(\frac{D m}{n}\right)
\end{aligned}
$$

with

$$
J_{s}(t)=\epsilon^{*}(m D-N t) \sum_{\substack{d \mid t \\(d, D)=1}} \epsilon(d) d^{1-2 s} \cdot \sum_{\delta^{\prime} \mid(D, t)} \epsilon_{\delta^{\prime}}(N t(N t-m D)) t_{\delta^{\prime}}^{1-2 s}
$$


where $\epsilon^{*}=\epsilon \cdot \epsilon_{D}^{-1}$ and $t_{\delta^{\prime}}$ denotes the maximal positive divisor of $t$ whose prime factors are those of $\delta^{\prime}$.

Further, we have the functional equation,

$$
\widetilde{\Phi}_{1-s, g}=\epsilon(-N)\left(D N^{-1}\right)^{2 s-1} \widetilde{\Phi}_{s, g} .
$$

Proof. By definition, we have

$$
\tilde{\phi}_{s, g}(m)=B_{s}(N) \frac{b(m)}{m^{s}}+2 \pi i^{k} T_{m, N}(s)
$$

with

$$
T_{m, N}(s)==\sum_{\delta \mid D} \frac{\delta^{1 / 2-s}}{2 \pi i^{\ell}} \sum_{n=1}^{\infty} \frac{b^{\delta}(n)}{n^{1-s}} S_{N}^{\delta}(m \delta-n) \tilde{I}_{s}\left(\frac{\delta m}{n}\right) .
$$

One precise computation of $B_{s}(N)$ will give the first term in the formula of Theorem 11.5. Replacing $n$ by $n / \delta^{\prime}$, interchanging the sums, and using the formula for $b^{\delta}$ (equation (11.4)), we have

$$
T_{m, N}(s)=\frac{D^{1 / 2-s} b(D)}{2 \pi i^{\ell} \kappa(D)} \sum_{\substack{n \geq 1 \\ m D \equiv n(\bmod N)}} \frac{b(n)}{n^{1-s}} J_{s}^{*}(n) \tilde{I}_{s}\left(\frac{D m}{n}\right)
$$

where

$$
J_{s}^{*}(n)=\sum_{\delta^{\prime} \mid(D, n)} \sqrt{\delta^{\prime}} \kappa\left(\delta^{\prime}\right) \epsilon_{\delta}\left(n / \delta^{\prime}\right) S_{N}^{\delta}\left(\frac{m D-n}{\delta^{\prime}}\right) .
$$

If $n=m D$, then

$$
J_{s}^{*}(m D)=\epsilon(m D) L(2 s-1, \epsilon) .
$$

Applying the functional equation of $L(s, \epsilon)$, the term $n=m D$ in the last formula of $T_{m, N}(s)$ will give the second term in the formula of the Theorem. Now we assume that $n \neq m D$. Then

$$
J_{s}^{*}(n)=\sum_{\delta^{\prime} \mid(D, n)} \sqrt{\delta^{\prime}} \kappa\left(\delta^{\prime}\right) \epsilon_{\delta}\left(n / \delta^{\prime}\right) S_{N}^{\delta}\left(\frac{m D-n}{\delta^{\prime}}\right) .
$$

Notice that $S_{N}^{\delta}$ is nonzero only if $m D \equiv n(\bmod N)$. Write $t=\frac{m D-n}{N}$, then

$$
S_{N}^{\delta}\left(N t / \delta^{\prime}\right)=\frac{G(\delta) \epsilon_{\delta^{\prime}}\left(N t / \delta^{\prime}\right)}{\left(\delta^{\prime}\right)^{2 s}\left(t_{\delta^{\prime}} / \delta^{\prime}\right)^{2 s-1}} \sum_{\substack{d \mid t \\(d, D)=1}} \epsilon(d) d^{1-2 s}
$$

Since $G(\delta)=\kappa\left(\delta^{\prime}\right) \sqrt{\delta^{\prime}}$, and

$$
\epsilon_{\delta^{\prime}}(-1) \epsilon_{\delta}\left(n / \delta^{\prime}\right) \epsilon_{\delta^{\prime}}\left(N t / \delta^{\prime}\right)=\epsilon^{*}(n) \epsilon_{\delta^{\prime}}(-n N t),
$$

we have $J_{s}^{*}(n)=J_{s}(t)$ as in the last term in the formula of Theorem 11.5.

We now obtain the functional equation for

$$
J_{s}(t)=\epsilon^{*}(m D-N t) \sum_{\substack{d \mid t \\(d, D)=1}} \epsilon(d) d^{1-2 s} \cdot \sum_{\delta^{\prime} \mid(D, t)} \epsilon_{\delta^{\prime}}(N t(N t-m D)) t_{\delta^{\prime}}^{1-2 s} .
$$


Replace $d$ by $|t| /\left(t_{D} d\right)$ in the first sum, and replace $\delta^{\prime}$ by $(D, t) / \delta^{\prime}$ in the second sum to obtain

$$
J_{1-s}(t)=t^{2 s-1} \epsilon_{D}\left(|t| / t_{D}\right) \epsilon_{(D, n)}(-N t n) J_{s}(t) .
$$

Notice that $\epsilon_{D}\left(|t| / t_{D}\right)=\operatorname{sgn}(t) \epsilon_{D}(t)$, and for any $p$ not dividing $(t, D)$,

$$
\epsilon_{p}(-N t n)=1
$$

because $-N t n \equiv(N t)^{2}(\bmod p)$. We, therefore, obtain that

$$
\epsilon\left(|t| / t_{D}\right) \epsilon_{(D, t)}(-N t n)=\operatorname{sgn}(t) \epsilon_{D}(t) \epsilon_{D}(-N t n)=\operatorname{sgn}(t) \epsilon(-N) \epsilon(n) .
$$

It follows that $J_{s}(t)$ satisfies the functional equation

$$
J_{1-s}(t)=t^{2 s-1} \operatorname{sgn}(t) \epsilon(-N) \epsilon(n) J_{s}(t) .
$$

Combining this with the functional equation for $\tilde{I}_{s}$ in Proposition 8.6, we obtain the functional equation for $\widetilde{\Phi}_{s, g}$.

REMARK. In the case that $g$ is a theta series attached to an imaginary quadratic field, and $m$ is prime to $N$, Gross and Zagier [G-Z] have computed the value (when $\epsilon(N)=1$ ) and the derivative (when $\epsilon(N)=-1$ ) of $\tilde{\phi}_{s, g}(m)$. It is not difficult to see that our results coincide with those of Gross-Zagier in this case. Our results go beyond [G-Z] in that we give the whole kernel (not only the special value or derivatives) in terms of divisor functions and hypergeometric functions.

\section{REFERENCES}

[G-Z] B. Gross And D. B. Zagier, Heegner points and derivatives of L-series, Invent. Math., 84 (1986), p. 225-320.

[L] Wen Ch'Ing WinNie LI, L-series of Rankin type and their functional equations, Math. Ann., 244:2 (1979), p. 135-166.

[Ra] S. RAMANUJAN, On certain trigonometrical sums and their applications in the theory of numbers, Trans. Cambridge Philos. Soc., XXII:13 (1918), p. 259-276.

[R] R. RANKIN, Contributions to the theory of Ramanujan's function $\tau(n)$ and similar arithmetic functions, I and II, Proc. Cambridge Phil. Soc., 35 (1939), p. 351-356, 357-372.

[Sa] P. Sarnak, Some applications of modular forms, in Cambridge Tracts in Math. 99, Cambridge Univ. Press, Cambridge, 1990, p. 22-25.

[S1] A. Selberg, Bemerkungen über eine Dirichletsche reihe, die mit der theorie der modulformer nahe verbunden ist, Arch. Math. Naturvid., 43 (1940), p. 47-50.

[S2] A. Selberg, On the estimation of Fourier coefficients of modular forms, in Proc. Sympos. Pure Math. (Cal Tech, Pasadena, Cal., 1963) VIII, Amer. Math. Soc., Providence, R.I., 1963 , p. $1-15$. 
